\title{
Clinical Outcomes of Patients with Advanced Solid Tumors of Cutaneous Capillary Hyperplasia Following Treatment with Camrelizumab: A Retrospective Case- Control Study
}

\author{
Wang $\mathrm{X}^{1}$, Long $\mathrm{Q}^{1}$, He $\mathrm{Q}^{1}$, Li J ${ }^{1}$, Su Y ${ }^{2}$, Zhao J Y** \\ and $\mathrm{Li} \mathrm{F}^{\mathbf{1} *}$ \\ ${ }^{1}$ Department of Oncology, The Second Affiliated Hospital \\ of Anhui Medical University, Hefei, Anhui Province, \\ China \\ ${ }^{2}$ Department of Ophthalmology, Anhui Provincial \\ Children's Hospital, Hefei 230000, Anhui Province, \\ China \\ ${ }^{3}$ Department of Pediatric Oncology Center, Beijing \\ Children Hospital, Capital Meidical University, National \\ Center for Children's Health, China \\ *Corresponding author: J unyang Zhao, Department \\ of Pediatric Oncology Center, Beijing Children Hospital, \\ Capital Meidical University, National Center for \\ Children's Health, China
}

Fanfan Li, Department of Oncology, The Second Affiliated Hospital of Anhui Medical University, Hefei 230022, Anhui Province, China

Received: J une 22, 2021; Accepted: July 15, 2021; Published: July 22, 2021

\begin{abstract}
Objective: To investigate the difference of clinical outcomes between patients with and without Reactive Cutaneous Capillary Endothelial Proliferation (RCCEP) after camrelizumab treatment. Methods: A retrospective, matched case-control study was designed. A total of 92 patients with advanced solid tumors treated with camrelizumab at xx hospital between July 2019 and October 2020 were included, of whom 16 patients developed RCCEP (RCCEP group) and the remaining 76 served as the control group. The primary endpoint is Progression-Free Survival (PFS), and the secondary endpoint is Objective Response Rate (ORR) and Overall Survival (OS). Multivariate Cox regression analysis is used to assess the relevant indicators of PFS.
\end{abstract}

Results: Compared with the control group, significantly increased ORR was observed in patients with RCCEP $(56.3 \%$ vs $19.7 \%)(P<0.05)$. The PFS was 13 months (5-15 months) in the RCCEP group and 6 months (2-11 months) in the control group. Compared with the control group, the PFS was significantly higher in the RCCEP group (HR=0.555, 95\% Cl: 0.278-0.985, $\mathrm{P}<0.05)$. In multivariate Cox regression, RCCEP remained statistically significant after excluding potential confounders ( $\mathrm{HR}=0.312,95 \% \mathrm{Cl}$ : 0.095-0.637, $\mathrm{P}<0.01)$ and was associated with PFS in patients.

Conclusion: In camrelizumab treatment, the occurrence of RCCEP may be a marker of strong immune response and improved tumor treatment outcomes, and has potential predictive value in patient efficacy and prognosis.

Keywords: Camrelizumab; Advanced tumors; Reactive cutaneous capillary endothelial proliferation; Prognosis

\section{Introduction}

Malignant tumors have become the primary disease threatening the life and health of all mankind [1]. In China, due to lifestyle changes and increasing aging and other factors, the incidence and mortality of malignant tumors are increasing rapidly every year, which leads to a significant increase in the number of patients with advanced malignant tumors [2]. Essentially all such patients develop metastases, and surgical treatment is of limited significance. Continued advances in chemotherapy, as well as targeted therapeutic approaches, have provided more promising treatment options for patients with advanced solid tumors [3,4].

Programmed Death receptor 1 (PD-1)/Programmed DeathLigand 1 (PD-L1) signaling pathway has been widely studied in clinical practice in recent years. As one of the key links of immune checkpoints in tumor immune escape, it plays an important role in tumor immune escape [5]. Currently, PD-1/PD-L1 inhibitors have been proven effective in cancer therapy and have recently been approved by the US Food and Drug Administration (FDA), and have been successfully used to treat a variety of solid tumor types [6,7].
Carrelizumab is a PD-1/PD-L1 inhibitor independently developed in China. Since its introduction, it has attracted much industry attention and taken the international stage many times, and has been widely recognized in the field of oncology at home and abroad. Camrelizumab is a humanized IgG4k-type anti-PD-1 monoclonal antibody that binds to PD-1 to block the PD-1/PD-L1 pathway, thereby activating $\mathrm{T}$ cells and producing sustained antitumor effects [8]. Since camrelizumab showed a good survival benefit in a singlearm phase II clinical trial of classical Hodgkin lymphoma, the drug was approved by the China Food and Drug Administration (CFDA) on May 29, 2019, for the treatment of relapsed or refractory classical Hodgkin lymphoma after at least second-line systemic chemotherapy [9]. Camrelizumab is also being studied for the treatment of various malignancies such as lung cancer, gastric cancer, nasopharyngeal carcinoma, as well as liver cancer [10].

We note that Reactive Cutaneous Capillary hyperplasia (RCCEP) appears to be a dermatological adverse event unique to camrelizumab treatment $[11,12]$. Even though dermatological adverse events are very common in PD-1/PD-L1 inhibitor drugs, RCCEP has not been reported in other PD-1/PD-L1 inhibitor treatments. The time to
Jis Markers - Volume 6 Issue 2 - 2021

ISSN : 2380-0682 | www.austinpublishinggroup.com

Wang et al. (C) All rights are reserved
Citation: Wang X, Long Q, He Q, Li J, Su Y, Zhao JY, et al. Clinical Outcomes of Patients with Advanced Solid Tumors of Cutaneous Capillary Hyperplasia Following Treatment with Camrelizumab: A Retrospective CaseControl Study. J Dis Markers. 2021; 6(2): 1044. 
appearance of RCCEP after camrelizumab treatment is in the range of 20 to $42 \mathrm{~d}[12,13]$, with a significant dose dependence, and the shorter the time to appearance [12]. It is usually scattered throughout the body and can also be clustered, with the head and neck, trunk, and extremities being the most common parts $[11,12]$. Histopathologically, RCCEP showed clustered proliferation of thin-walled vessels in the dermis, vascular congestion and dilatation, and focal with a tendency to thrombosis [8]. The specific pathogenesis by which camrelizumab treatment leads to RCCEP is currently unknown. Some scholars Chen et al. [12] believe that this can be related to the imbalance between angiogenic contributors and inhibitors. Additional studies [14] have found that camrelizumab has off-target binding specificity, so it may promote angiogenesis and hemangioma formation by up-regulating Vascular Endothelial Growth Factor Receptor 2 (VEGFR2).

One phenomenon of interest is that there appears to be an association between RCCEP onset and higher response after camrelizumab treatment. In a previous single-arm multicenter phase II study of Song et al. [11] camrelizumab in relapsed or refractory classical Hodgkin's lymphoma, 97.3\% of patients developed RCCEP while achieving an Objective Response Rate (ORR) of 76.0\%. A recent prospective phase I observational study [15] involving 98 patients with advanced solid tumors also found that after camrelizumab treatment, patients experienced grade 1-2 RCCEP, while signs of significant tumor regression were observed. However, whether RCCEP in camrelizumab treatment is associated with a higher shortterm response as well as long-term prognosis in patients has not been well characterized.

Because it acts by reactivating the antitumor $\mathrm{T}$ cell response, and hyperactivation of the immune system may contribute to a series of immune-related adverse events $[5,16,17]$, such as RCCEP. It is, therefore, reasonable to assume that RCCEP onset may be a sign of a more robust immune antitumor response. In this study, we conducted a retrospective case-control study and assessed the relationship between RCCEP onset after camrelizumab treatment and multiple oncological outcomes, such as Objective Response Rate (ORR), Progression-Free Survival (PFS), and/or Overall Survival (OS), in patients with advanced solid tumors.

\section{Material and Methods}

\section{Patients}

A total of 92 patients with advanced solid tumors who developed RCCEP while receiving camrelizumab in our hospital between July 2019 and October 2020 were included in the study (RCCEP group). Inclusion criteria: 1) All patients developed RCCEP within 3 months after receiving camrelizumab treatment and were diagnosed by dermoscopy and tissue case examination; 2) Patients had complete treatment and follow-up data. Exclusion criteria: 1) Patients with a history of RCCEP at the time of treatment; 2) Patients with RCCEP due to other causes. Matched controls for RCCEP patients were based on age, gender, and camrelizumab treatment cycle, with a maximum of 5 controls matched for each case. And the control group were all patients who mentioned the onset of RCCEP after starting camrelizumab treatment until their progression was recorded.

\section{Efficacy Assessments}

Best overall response (BOR): Determine the best response data for patients treated with camrelizumab by manually examining patient cases. These include a Complete Response (CR), Partial Response (PR), Stable Disease (SD), and Progressive Disease (PD). PD was defined as: (1) an increase of more than $20 \%$ in the diameter of a patient's original tumor lesion and or the appearance of a new tumor lesion, and (2) the occurrence of death in a patient. Overall response rate (ORR) was defined as the percentage of patients with $\mathrm{CR}$ and PR. Disease control rate (DCR) was defined as the percentage of patients with CR, PR, and SD.

Progression-free survival (PFS): Is defined as the time interval from the start of treatment with camrelizumab to the occurrence of disease progression or death. Overall Survival (OS) was defined as the interval from the start of treatment with camrelizumab to the occurrence of death from any cause.

\section{Statistical analysis}

SPSS version 23.0 (SPSS, Inc., Chicago, IL, USA) software was applied for statistical analysis. Enumeration data were expressed as a case (percentage) [n (\%)], $\chi 2$ test or Fisher exact test was performed; patient age conformed to the normal distribution, expressed as mean \pm standard deviation $(\bar{X} \pm S)$, and independent sample t-test was used for comparison. Camrelizumab treatment weeks were expressed as median (range), and the Mann-Whitney $U$ test was used for comparison. Kaplan-Meier curves for PFS in the two groups were plotted using GraphPad 7.0 and compared using the log-rank test; multivariate Cox regression analysis was used to evaluate factors influencing PFS in patients. The test level was $\alpha=0.05$, and $\mathrm{P}<0.05$ was considered statistically significant.

\section{Results}

\section{Baseline data of patients in the two groups}

Of the 92 patients with advanced solid tumors treated with camrelizumab, 16 patients were eligible for the study. They were all diagnosed with RCCEP by dermoscopy and had no history of RCCEP in previous cases. Propensity matching was performed in the remaining patients according to age, gender, and timing of camrelizumab treatment, and 76 patients were included as a control group (none of the patients had RCCEP). The sociodemographic statistics and clinical data of the patients in the two groups are shown in Table 1. Only a few patients in both groups were treated with camrelizumab. Due to sample size limitations, not all cancer types were involved in the study. Among them, lung cancer and digestive tract tumors accounted for the majority (Table 2). Also, we noted a significant incidence of RCCEP for lung cancer (30.8\% vs 7.7\%) compared with digestive tract tumors $(\mathrm{P}=0.027)$.

\section{Efficacy}

All 92 patients with advanced solid tumors were evaluated for a response during follow-up through december 30, 2020. The ORR was $26.1 \%$ (including $6 \mathrm{CR}$ and $18 \mathrm{PR}$ ) and the DCR was $75.0 \%$ (including $6 \mathrm{CR}, 18 \mathrm{PR}$, and $45 \mathrm{SD}$ ) with camrelizumab treatment. A total of 53 PFS events were reported, with a PFS of 7 months (range, 2 to 15 months) (Figure 1). Twenty-one patients died, with an OS of 3 months and a maximum of 15 months, and the OS has not been reached.

\section{RCCEP patients had higher ORR and PFS}

A significant increase in ORR (56.3\% vs 19.7\%) was observed in 
Table 1: Comparison of baseline data between the two groups.

\begin{tabular}{|c|c|c|c|}
\hline Item & $\begin{array}{l}\text { RCCEP group } \\
(n=16)\end{array}$ & $\begin{array}{c}\text { Control group } \\
(n=76)\end{array}$ & $\mathbf{P}$ \\
\hline Mean age (years, $\bar{x} \pm s$ ) & $58.9 \pm 11.4$ & $60.3 \pm 9.9$ & 0.163 \\
\hline Gender, n (\%) & & & 0.82 \\
\hline Male & $12(75.0)$ & $59(77.6)$ & \\
\hline Female & $4(25.0)$ & $17(22.4)$ & \\
\hline $\begin{array}{l}\text { Camrelizumab treatment weeks, } \\
\text { median (range) }\end{array}$ & $13(4-30)$ & $15(2-35)$ & 0.293 \\
\hline Camrelizumab treatment, n (\%) & $1(6.3)$ & $10(13.2)$ & 0.439 \\
\hline Number of metastatic sites, $\mathrm{n}(\%)$ & & & $0.667^{*}$ \\
\hline 0 & $0(0.0)$ & $7(9.2)$ & \\
\hline 1 & $8(50.0)$ & $31(40.8)$ & \\
\hline 2 & $4(25.0)$ & $23(30.3)$ & \\
\hline$\geq 3$ & $4(25.0)$ & $15(19.7)$ & \\
\hline \multicolumn{4}{|l|}{ Metastatic site, n (\%) } \\
\hline Central nervous system & $1(6.3)$ & $7(9.2)$ & 0.703 \\
\hline Lung & $9(56.3)$ & $24(31.6)$ & 0.061 \\
\hline Liver & $3(18.8)$ & $22(29.0)$ & 0.405 \\
\hline Bone & $7(43.8)$ & $20(26.3)$ & 0.164 \\
\hline Lymph nodes & $7(43.8)$ & $41(54.0)$ & 0.458 \\
\hline Other & $3(18.8)$ & $18(23.7)$ & 0.669 \\
\hline
\end{tabular}

Table 2: RCCEP incidence by tumor type after camrelizumab treatment.

\begin{tabular}{|l|c|c|}
\hline \multicolumn{1}{|c|}{ Tumor type } & Patients, $\mathbf{n}$ & $\begin{array}{c}\text { Incidence of RCCEP, } \\
\mathbf{n}(\%)\end{array}$ \\
\hline Lung cancer & 39 & $12(30.8)$ \\
\hline Small cell lung cancer & 6 & $1(2.6)$ \\
\hline Lung adenocarcinoma & 18 & $7(17.9)$ \\
\hline Squamous cell carcinoma of lung & 15 & $4(10.3)$ \\
\hline Alimentary tract & 26 & $2(7.7)$ \\
\hline Colorectal Cancer & 5 & $0(0.0)$ \\
\hline Gastric Cancer & 13 & $1(3.8)$ \\
\hline Esophageal squamous cell & 8 & $1(3.8)$ \\
\hline carcinoma & 10 & $1(10.0)$ \\
\hline Liver cancer & 11 & $0(0.0)$ \\
\hline Urogenital System & 3 & $0(0.0)$ \\
\hline Kidney cancer & 2 & $0(0.0)$ \\
\hline Ureteral urothelial carcinoma & 3 & $0(0.0)$ \\
\hline Ovarian cancer & 3 & $0(0.0)$ \\
\hline Cervical cancer & 1 & $0(0.0)$ \\
\hline Laryngeal squamous cell carcinoma & 4 & $1(25.0)$ \\
\hline Nasopharyngeal carcinoma & & $0.0)$ \\
\hline Osteosarcoma & 1 & \\
\hline
\end{tabular}

patients with RCCEP compared with controls $(\mathrm{P}<0.05)$ (Table 3). PFS was 13 months (5-15 months) in the RCCEP group and 6 months (2-11 months) in the control group (Figure 2). Compared with the control group, PFS was significantly higher in patients with RCCEP $(\mathrm{HR}=0.555,95 \% \mathrm{CI}: 0.278-0.985, \mathrm{P}<0.05)$. OS had not been reached in both groups, and as of the follow-up by October 30, 2020, OS was $87.5 \%$ in the RCCEP group and $72.4 \%$ in the control group.
Table 3: Response in the two groups, $\mathrm{n}(\%)$

\begin{tabular}{|c|c|c|c|c|c|c|}
\hline Group & N & CR & PR & SD & PD & ORR (\%) \\
\hline RCCEP Group & 16 & $4(25.0)$ & $5(31.3)$ & $4(25.0)$ & $3(18.8)$ & 56.3 \\
\hline Control group & 76 & $2(2.6)$ & $13(17.1)$ & $41(44.0)$ & $20(26.3)$ & 19.7 \\
\hline X2 & \multicolumn{7}{|r}{} & & 9.139 \\
\hline P & \multicolumn{1}{|r}{} & & 0.003 \\
\hline
\end{tabular}

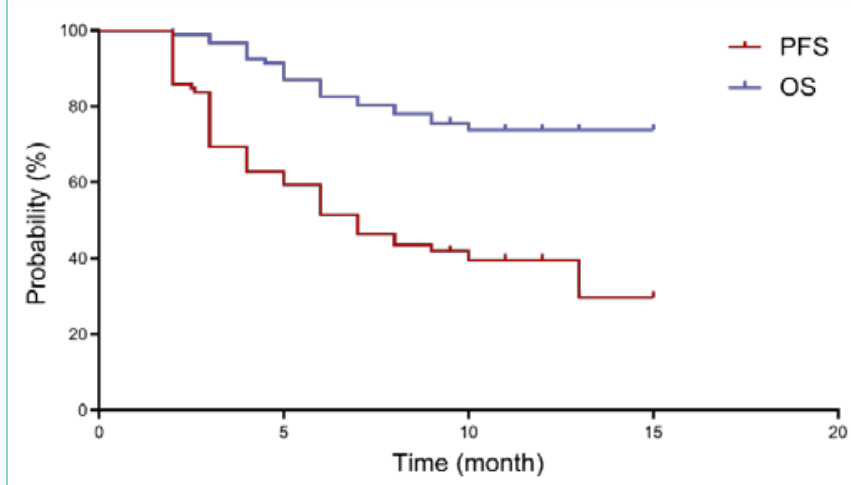

Figure 1: Kaplan-Meier curves of PFS and OS for all patients.

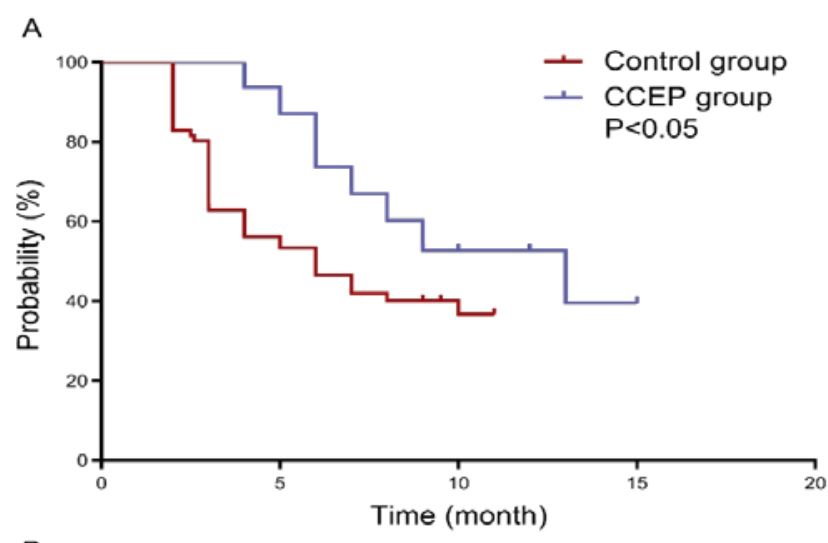

B

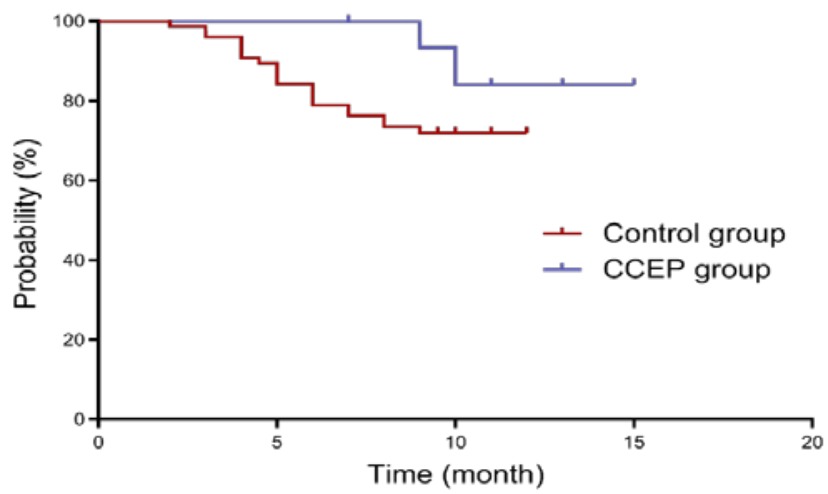

Figure 2: Kaplan-Meier curves of PFS and OS in the two groups. A, PFS was significantly increased in the RCCEP group (13 months vs 6 months) compared with the control group $(\mathrm{HR}=0.555,95 \% \mathrm{Cl}$ : 0.278-0.985, $\mathrm{P}<0.05)$ $\mathrm{B}$, OS had not been reached in the two groups, and there was no significant difference $(P>0.05)$.

COX regression analysis shows that RCCEP is associated with PFS

The results of univariate analysis showed that camrelizumab 

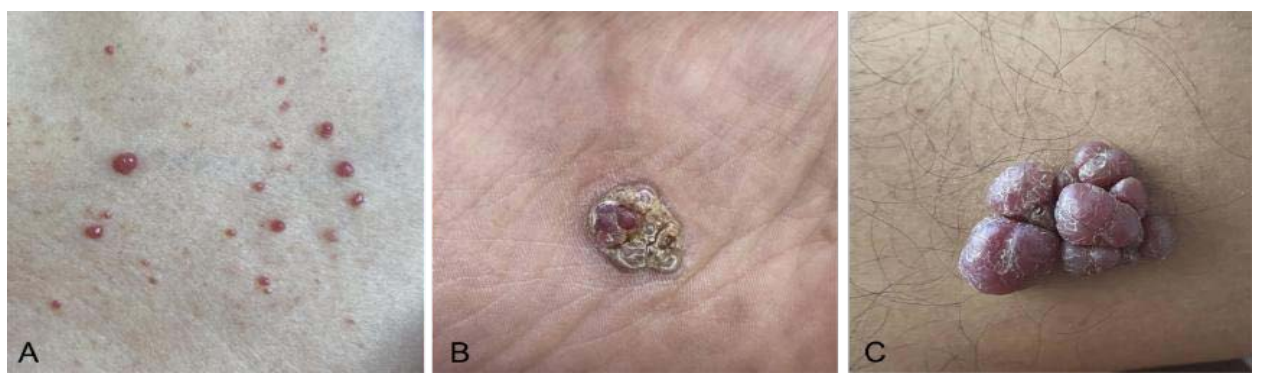

Figure 3: Photographs of RCCEP cases. A, Level 1 RCCEP. B, Grade 2 RCCEP, resolving. C, Level 2 RCCEP, plateau phase.

Table 4: Univariate and multivariate Cox regression analysis of progression free survival (PFS) ( $n=92,53$ events)

\begin{tabular}{|c|c|c|c|c|c|c|}
\hline \multirow{2}{*}{ Variable } & \multicolumn{3}{|c|}{ Univariate analysis } & \multicolumn{3}{|c|}{ Multivariate analysis } \\
\hline & $H R$ & $95 \% \mathrm{Cl}$ & $P$ & $H R$ & $95 \% \mathrm{Cl}$ & $P$ \\
\hline Age (per 1 year) & 0.993 & 0.768 to 1.195 & 0.306 & & & \\
\hline Gender (male vs female) & 1.063 & 0.954 to 1.288 & 0.522 & & & \\
\hline Camrelizumab treatment (yes vs no) & 1.665 & 1.116 to 2.092 & 0.026 & 1.723 & 1.216 to 2.435 & 0.015 \\
\hline Number of metastatic sites ( $\leq 2$ vs $>2$ ) & 0.702 & 0.592 to 0.936 & 0.013 & 0.892 & 0.647 to 1.152 & 0.208 \\
\hline Metastases to nervous system (yes vs no) & 1.315 & 0.973 to 1.825 & 0.177 & & & \\
\hline Visceral metastasis (yes vs no) & 1.202 & 0.902 to 1.636 & 0.295 & & & \\
\hline Tumor type (lung cancer vs other tumors) & 0.805 & 0.319 to 0.969 & 0.02 & 0.805 & 0.519 to 1.106 & 0.096 \\
\hline Incidence of RCCEP (yes vs no) & 0.653 & 0.109 to 0.803 & 0.018 & 0.312 & 0.095 to 0.637 & 0.006 \\
\hline
\end{tabular}

Important $\mathrm{P}$ values are shown in bold.

treatment $(\mathrm{HR}=1.665,95 \% \mathrm{CI}: 1.116-2.092, \mathrm{P}<0.05)$ was associated with shorter PFS. In contrast, the number of metastatic sites $\leq 2$, lung cancer, and the incidence of RCCEP were associated with PFS ( $\mathrm{HR}=0.702,0.805,0.653$, respectively). By multivariate analysis, the incidence of RCCEP ( $\mathrm{HR}=0.312,95 \% \mathrm{CI}$ : 0.095-0.637, $\mathrm{P}<0.01$ ) remained statistically significant after excluding potential mixes. In addition, treatment with camrelizumab was still associated with shorter PFS (HR=1.723, 95\% CI: 1.216-2.435, $\mathrm{P}<0.05$ ) (Table 4).

\section{Treatment of RCCEP}

All 16 patients had grade 1-2 RCCEP (Figure 3), and only 6.3\% interrupted camrelizumab treatment and resumed treatment after 2 weeks. All patients were asked to avoid scratching or rubbing the rash site, along with appropriate protection and follow-up observation. Mainly pay attention to its growth rate and nodule surface changes. Patients who developed ulceration were given external application of Yunnan Baiyao powder and antibiotic ointment to prevent infection if necessary. There were no cases of reduction of camrelizumab single dose due to RCCEP.

\section{Discussion}

The current study primarily assessed the relationship between RCCEP onset after camrelizumab treatment and ORR, PFS, and OS in patients with advanced solid tumors. The study results showed that patients with RCCEP had a significantly higher ORR (56.3\% vs 19.7\%) and a significantly increased PFS (13 months $v s 6$ months) compared with patients without RCCEP (HR $=0.555$, 95\% CI: $0.278-0.985)$. Due to limited follow-up, OS was not reached in both groups. We further analyzed the relationship between RCCEP and PFS by Cox regression, and the results showed that RCCEP was associated with PFS in patients after excluding potential confounders
(HR=0.312, 95\% CI: 0.095-0.637). This suggests that the onset of RCCEP after camrelizumab treatment may lead to multiple favorable tumor outcomes in patients with advanced solid tumors, including ORR, PFS, which may have practical clinical value for the prediction of clinical efficacy in patients. A recent study [12] also found that patients who developed RCCEP after camrelizumab treatment achieved an objective response rate of $28.9 \%$, while patients without RCCEP did not respond to treatment. Future prospective studies will further confirm the predictive value of RCCEP as a treatment with camrelizumab for a strong antitumor response and leading to improved tumor outcomes.

Previous reports have shown that RCCEP is common after camrelizumab treatment, and the incidence of treatment is as high as $66.8 \%$ to $97.3 \%$ [18-20]. However, it was found that the incidence of RCCEP was significantly reduced when camrelizumab was combined with other drugs, for example, in combination with FOLFOX4 regimen or apatinib, especially in combination with apatinib [15]. A study [21] reported that the incidence of RCCEP was $12.1 \%$ in camrelizumab $200 \mathrm{mg}+$ apatinib for hepatocellular carcinoma, gastric, or esophagogastric junction cancer. In other studies of camrelizumab in combination with gemcitabine and cisplatin in nasopharyngeal carcinoma [22,23], the incidence of RCCEP was slightly higher at $20 \%$. In this study, $91.3 \%$ (8/92) of patients were treated with camrelizumab combined with other drug regimens, and the incidence of RCCEP was $17.4 \%$ (16 of 92). This compares with previous reports that most of the tumors in this study were lung cancer and digestive tract tumor types, and the incidence of RCCEP was overall in line with the range of incidence of RCCEP when camrelizumab was combined with other drugs as previously reported $[15,23,24]$. 
Regarding the treatment of RCCEP after camrelizumab treatment, there is no effective prevention in clinical practice. However, in the current reports, RCCEP is basically graded 1 to 2 . Even in the study by Song et al. [11], $97.3 \%$ of RCCEPs were within grade 2 . There were also no patients in our study who exceeded grade 2. And RCCEP mostly involves the skin and does not involve the viscera. Even the occurrence of ruptured bleeding or infection after ulceration does not endanger the patient's life [25]. Alternatively, current studies [11,26] show that RCCEP is self-limiting and has a favorable prognosis. The spontaneous resolution was observed immediately during treatment and the complete resolution was observed after discontinuation of the drug, with a time of about 53.5 days [12]. And no cases of recurrence and deterioration have been reported. Treatment, continued camrelizumab treatment is recommended for RCCEPs without more than grade 2, and it should be emphasized to pay attention to careful observation and avoid scratching or rubbing, rather than aggressive treatment [11]. For patients with a maximum diameter $>10 \mathrm{~mm}$, local excision surgery or laser therapy can be performed as needed [27] Patients with ulceration can be local anti-inflammatory, prevention of infection.

Limitations of this study include its retrospective nature, and the reliability of the data relies heavily on the completeness and accuracy of case records and follow-up data. Also, due to sample size limitations, not all solid tumor types could be involved. Future prospective studies will help to reduce the bias of the data. Also, larger studies will allow the assessment of each type of tumor.

In summary, the current study suggests that the development of RCCEP after camrelizumab treatment in advanced solid tumors may be a marker of robust immune response and improved tumor treatment outcomes, and conversely, if RCCEP does not occur, it may help clinical identification of patients with poor response to camrelizumab treatment and then change treatment regimens. The value of RCCEP in the potential prediction of patient efficacy and prognosis deserves further in-depth study.

\section{References}

1. Ren H, Liu C, Wang R, et al. Core Competencies Required for Gastroenterology Nursing Specialists in China. Gastroenterol Nurs. 2019; 42: 169-178.

2. Chen W, Zheng R, Baade PD, et al. Cancer statistics in China, 2015. CA Cancer J Clin. 2016; 66: 115-132.

3. Gotwals P, Cameron S, Cipolletta D, et al. Prospects for combining targeted and conventional cancer therapy with immunotherapy. Nat Rev Cancer. 2017; 17: 286-301.

4. Nagasaka M, Gadgeel SM. Role of chemotherapy and targeted therapy in early-stage non-small cell lung cancer. Expert Rev Anticancer Ther. 2018; 18: $63-70$.

5. Umansky V, Blattner C, Fleming V, et al. Myeloid-derived suppressor cells and tumor escape from immune surveillance. Semin Immunopathol. 2017; 39: $295-305$

6. Ilie M, Hofman P. Atezolizumab in advanced non-small cell lung cancer. J Thorac Dis. 2017; 9: 3603-3606.

7. Hamid O, Robert C, Daud A, et al. Safety and tumor responses with lambrolizumab (anti-PD-1) in melanoma. N Engl J Med. 2013; 369: 134-144.

8. Nie J, Wang C, Liu Y, et al. Addition of Low-Dose Decitabine to AntiPD-1 Antibody Camrelizumab in Relapsed/Refractory Classical Hodgkin Lymphoma. J Clin Oncol. 2019; 37: 1479-1489.

9. Markham A, Keam SJ. Camrelizumab: First Global Approval. Drugs. 2019;
79: $1355-1361$.

10. Wei Z, Yang X, Ye X, et al. Camrelizumab combined with microwave ablation improves the objective response rate in advanced non-small cell lung cancer. J Cancer Res Ther. 2019; 15: 1629-1634.

11. Song Y, Wu J, Chen X, et al. A Single-Arm, Multicenter, Phase II Study of Camrelizumab in Relapsed or Refractory Classical Hodgkin Lymphoma. Clin Cancer Res. 2019; 25: 7363-7369.

12. Chen X, Ma L, Wang X, et al. Reactive capillary hemangiomas: a nove dermatologic toxicity following anti-PD-1 treatment with SHR-1210. Cancer Biol Med. 2019; 16: 173-181.

13. Mo H, Huang J, Xu J, et al. Safety, anti-tumour activity, and pharmacokinetics of fixed-dose SHR-1210, an anti-PD-1 antibody in advanced solid tumours: a dose-escalation, phase 1 study. Br J Cancer. 2018; 119: 538-545.

14. Song $\mathrm{Y}$, Gao Q, Zhang $\mathrm{H}$, et al. Treatment of relapsed or refractory classical Hodgkin lymphoma with the anti-PD-1, tislelizumab: results of a phase 2 , single-arm, multicenter study. Leukemia. 2020; 34: 533-542.

15. Teng Y, Guo R, Sun J, et al. Reactive capillary hemangiomas induced by camrelizumab (SHR-1210), an anti-PD-1 agent. Acta Oncol. 2019; 58: 388389.

16. Postow MA, Sidlow R, Hellmann MD. Immune-related adverse events associated with immune checkpoint blockade. New England Journal of Medicine. 2018; 378: 158-168.

17. Brahmer JR, Lacchetti C, Schneider BJ, et al. Management of immunerelated adverse events in patients treated with immune checkpoint inhibitor therapy: American Society of Clinical Oncology Clinical Practice Guideline. Journal of clinical oncology: official journal of the American Society of Clinical Oncology, 2018, 36(17): 1714

18. Qin S, Ren Z, Meng Z, et al. LBA27 A randomized multicentered phase II study to evaluate SHR-1210 (PD-1 antibody) in subjects with advanced Hepatocellular Carcinoma (HCC) who failed or intolerable to prior systemic treatment. Annals of Oncology. 2018; 29.

19. Huang J, Mo H, Zhang W, et al. Promising efficacy of SHR-1210, a nove anti-programmed cell death 1 antibody, in patients with advanced gastric and gastroesophageal junction cancer in China. Cancer. 2019; 125: 742-749.

20. Huang J, Xu B, Mo H, et al. Safety, activity, and biomarkers of SHR-1210, an anti-PD-1 antibody, for patients with advanced esophageal carcinoma. Clinical Cancer Research. 2018; 24: 1296-1304.

21. Xu J, Zhang Y, Jia R, et al. Anti-PD-1 Antibody SHR-1210 Combined with Apatinib for Advanced Hepatocellular Carcinoma, Gastric, or Esophagogastric Junction Cancer: An Open-label, Dose Escalation and Expansion Study. Clin Cancer Res. 2019; 25: 515-523.

22. Fang W, Yang Y, Ma Y, et al. Camrelizumab (SHR-1210) alone or in combination with gemcitabine plus cisplatin for nasopharyngeal carcinoma: results from two single-arm, phase 1 trials. Lancet Oncol. 2018; 19: 13381350.

23. Lv J-W, Li J-Y, Luo L-N, et al. Comparative safety and efficacy of antiPD-1 monotherapy, chemotherapy alone, and their combination therapy in advanced nasopharyngeal carcinoma: findings from recent advances in landmark trials. Journal for immunotherapy of cancer. 2019; 7: 159.

24. Xu J, Zhang $Y$, Jia R, et al. Anti-PD-1 antibody SHR-1210 combined with apatinib for advanced hepatocellular carcinoma, gastric, or esophagogastric junction cancer: an open-label, dose escalation and expansion study. Clinical Cancer Research. 2019; 25: 515-523.

25. Li W, Wei Z, Yang X, et al. Salvage therapy of reactive capillary hemangiomas: Apatinib alleviates the unique adverse events induced by camrelizumab in non-small cell lung cancer. J Cancer Res Ther. 2019; 15: 1624-1628.

26. Li L, Li G, Rao B, et al. Landscape of immune checkpoint inhibitor-related adverse events in Chinese population. Sci Rep. 2020; 10: 15567.

27. Yu Q, Wang WX. Camrelizumab (SHR-1210) leading to reactive capillary hemangioma in the gingiva: A case report. World J Clin Cases. 2020; 8: 624629. 